TRANSACTIONS OF THE

AMERICAN MATHEMATICAL SOCIETY

Volume 356, Number 5, Pages 1727-1749

S 0002-9947(03)03430-5

Article electronically published on December 9, 2003

\title{
COMMUTING TOEPLITZ OPERATORS ON THE POLYDISK
}

\author{
BOO RIM CHOE, HYUNGWOON KOO, AND YOUNG JOO LEE
}

\begin{abstract}
We obtain characterizations of (essentially) commuting Toeplitz operators with pluriharmonic symbols on the Bergman space of the polydisk. We show that commuting and essential commuting properties are the same for dimensions bigger than 2, while they are not for dimensions less than or equal to 2. Also, the corresponding results for semi-commutators are obtained.
\end{abstract}

\section{INTRODUCTION}

Let $D$ be the unit disk in the complex plane $\mathbb{C}$. For a fixed positive integer $n$, the unit polydisk $D^{n}$ is the cartesian product of $n$ copies of $D$. Let $L^{p}=L^{p}\left(D^{n}\right)$ denote the usual Lebesgue space with respect to the volume measure $V$ on $D^{n}$ normalized to have total mass 1. The Bergman space $A^{2}$ is then the closed subspace of $L^{2}$ consisting of all holomorphic functions on $D^{n}$. Let $P$ be the Bergman projection from $L^{2}$ onto $A^{2}$. For a function $u \in L^{\infty}$, the Toeplitz operator $T_{u}$ with symbol $u$ is defined by

$$
T_{u} f=P(u f)
$$

for $f \in A^{2}$. It is clear that $T_{u}: A^{2} \rightarrow A^{2}$ is a bounded linear operator.

In this paper we consider the problem of when two Toeplitz operators with pluriharmonic symbols commute or essentially commute. Recall that a complexvalued function $u \in C^{2}\left(D^{n}\right)$ is said to be pluriharmonic if its restriction to an arbitrary complex line that intersects $D^{n}$ is harmonic as a function of single complex variable. So, the notions of harmonicity and pluriharmonicity coincide on $D$. It turns out that every pluriharmonic function on $D^{n}$ can be expressed, uniquely up to an additive constant, as the sum of a holomorphic function and an antiholomorphic function. See Chapter 2 of [7] for details. Also, recall that two bounded linear operators $S_{1}, S_{2}$ on a Hilbert space $X$ are said to be essentially commuting on $X$ if the commutator $S_{1} S_{2}-S_{2} S_{1}$ is compact on $X$.

The problem of characterizing commuting Topelitz operators has been studied on various settings. Axler and Cučković 1] first obtained a complete description of harmonic symbols of commuting Toeplitz operators on D: If two Toeplitz operators with harmonic symbols commute, then either both symbols are holomorphic, or both symbols are antiholomorphic, or a nontrivial linear combination of the symbols is constant (the converse implication is also true and trivial). Later, some extensions of this characterization were obtained on higher-dimensional balls as in [3], [9] or

Received by the editors December 13, 2001.

2000 Mathematics Subject Classification. Primary 47B35; Secondary 32A36.

Key words and phrases. Toeplitz operator, $n$-harmonic function, Bergman space.

This work was supported by the Korea Research Foundation Grant (KRF-2000-DP0014).

(C)2003 American Mathematical Society 
[17]. Also, the same problem was considered on the annulus [6] and for more general symbols [2]. For related results on the (pluri)harmonic Bergman space, see [5] and [10].

For essentially commuting Toeplitz operators, Stroethoff [13] obtained characterizations of harmonic symbols on $D$. Choe and Lee 44 extended the result of Stroethoff to pluriharmonic symbols on the ball. On the other hand, the polydisk case was studied by Sun and Zheng [14. However, Sun and Zheng considered holomorphic or antiholomorphic symbols only. They proved that given $f, g \in H^{\infty}$, the following three conditions are equivalent for $n>1$ : (i) $T_{f}$ and $T_{\bar{g}}$ are commuting, (ii) $T_{f}$ and $T_{\bar{g}}$ are essentially commuting, (iii) for each $j$, either $\partial_{j} f=0$ or $\partial_{j} g=0$. Here, $H^{\infty}$ denotes the class of bounded holomorphic functions on $D^{n}$ and $\partial_{j}$ denotes the partial differential operator with respect to the $j$-th variable.

Our results obtained in this paper are characterizations of general pluriharmonic symbols of commuting or essentially commuting Toeplitz operators. For $n \geq 3$, as in the result of Sun and Zheng mentioned above, our results show that the commuting property and the essential commuting property are the same for Toeplitz operators with pluriharmonic symbols. However, they are different for $n=2$. Our method, whose main idea is adapted from 4, is entirely different from that of Sun and Zheng.

Following [11], we say that a complex-valued function $u \in C^{2}\left(D^{n}\right)$ is $n$-harmonic if $u$ is harmonic in each variable separately. More explicitly, $u$ is $n$-harmonic if

$$
\partial_{j} \bar{\partial}_{j} u=0, \quad j=1,2, \ldots, n \text {. }
$$

For a characterization of pluriharmonic symbols of commuting Toeplitz operators, we have the following. In what follows $H\left(D^{n}\right)$ denotes the class of all holomorphic functions on $D^{n}$.

Theorem 1.1. Let $u, v \in L^{\infty}$ be pluriharmonic symbols and assume $u=f+\bar{g}$, $v=h+\bar{k}$ for some $f, g, h, k \in H\left(D^{n}\right)$. Then the following statements are equivalent:

(a) $T_{u} T_{v}=T_{v} T_{u}$ on $A^{2}$.

(b) $f \bar{k}-h \bar{g}$ is n-harmonic.

In addition, we obtain characterizations of functions $f, g, h, k \in H\left(D^{n}\right)$ for which $f \bar{k}-h \bar{g}$ is $n$-harmonic. Before stating our result, we first introduce some notation. Let $I=\{1,2, \ldots, n\}$. For $J \subset I$, we write $H(J)$ for the set of all holomorphic functions independent of variables $z_{j}$ with $j \in I \backslash J$. Also, for $J_{1} \subset J_{2} \subset I$, we write $H\left(J_{2}\right) / H\left(J_{1}\right)$ for the set of all holomorphic functions in $H\left(J_{2}\right)$ whose power series (at the origin) do not contain any nonzero terms in $H\left(J_{1}\right)$.

Theorem 1.2. Let $f, g, h, k \in H\left(D^{n}\right)$. Then the following statements are equivalent:

(a) $f \bar{k}-h \bar{g}$ is n-harmonic.

(b) There are pairwise disjoint sets $I_{0}, \ldots, I_{m}$ with $\bigcup_{j=0}^{m} I_{j}=I$ for some nonnegative integer $m \leq n$, functions $f_{0}, h_{0}, g_{0}, k_{0}, p_{1}, \ldots, p_{m}, q_{1}, \ldots, q_{m}$ holomorphic on $D^{n}$, and constants $\alpha_{1}, \ldots, \alpha_{m}$ with the following properties:

(b1) $f_{0}, h_{0}, g_{0}, k_{0} \in H\left(I_{0}\right)$ and $p_{j}, q_{j} \in H\left(I_{0} \cup I_{j}\right) / H\left(I_{0}\right)$ for each $j$. 
(b2) We have

$$
\begin{array}{ll}
f=f_{0}+\sum_{j=1}^{m} p_{j}, \quad h=h_{0}+\sum_{j=1}^{m} \alpha_{j} p_{j}, \\
g=g_{0}+\sum_{j=1}^{m} q_{j}, \quad k=k_{0}+\sum_{j=1}^{m} \bar{\alpha}_{j} q_{j} .
\end{array}
$$

(b3) For each $r \in I_{0}$, one of the following four cases holds:

(i) $\partial_{r} f_{0}=\partial_{r} h_{0}=0$ and $\partial_{r} p_{j}=0$ for all $j$.

(ii) $\partial_{r} k_{0}=\partial_{r} g_{0}=0$ and $\partial_{r} q_{j}=0$ for all $j$.

(iii) $\partial_{r} f_{0}=\partial_{r} g_{0}=0$ and $\partial_{r} p_{j}=\partial_{r} q_{j}=0$ for all $j$.

(iv) $\partial_{r} k_{0}=\partial_{r} h_{0}=0$ and $\partial_{r} p_{j}=\partial_{r} q_{j}=0$ for all $j$.

(c) There are subsets $J_{1}, \ldots, J_{\ell}$ of $I$ for some integer $\ell \geq 1$, and holomorphic functions $A_{1}, \ldots, A_{\ell}, B_{1}, \ldots, B_{\ell}$ with $A_{i} \in H\left(J_{i}\right)$ and $B_{i} \in H\left(I \backslash J_{i}\right)$ for each $i$ such that

$$
f \bar{k}-h \bar{g}=\sum_{i=1}^{\ell} A_{i} \bar{B}_{i}
$$

Our next result is the essential version of Theorem 1.1 To state it, we need some more notation. First, we let

$$
\widetilde{\Delta}_{j} u(z)=\left(1-\left|z_{j}\right|^{2}\right)^{2} \partial_{j} \bar{\partial}_{j} u(z)
$$

for $j=1, \ldots, n$ and $u \in C^{2}\left(D^{n}\right)$. Here and elsewhere, $z_{j}$ denotes the $j$-th component of $z \in D^{n}$. Note that $u$ is $n$-harmonic if and only if $u$ is annihilated by all $\widetilde{\Delta}_{j}$. Thus, we will say that $u$ is boundary $n$-harmonic if $\lim _{a \rightarrow \partial D^{n}} \widetilde{\Delta}_{j} u(a)=0$ for all $j$. Here, $\partial D^{n}$ denotes the topological boundary of $D^{n}$. Also, we let $\Phi$ denote a class of functions related to the maximal ideal space of $H^{\infty}$. See Section 2 for the precise definition.

Theorem 1.3. Let $u, v \in L^{\infty}$ be pluriharmonic symbols and assume $u=f+\bar{g}$, $v=h+\bar{k}$ for some $f, g, h, k \in H\left(D^{n}\right)$. Then the following statements are equivalent:

(a) $T_{u}$ and $T_{v}$ are essentially commuting on $A^{2}$.

(b) $T_{u \circ \varphi} T_{v \circ \varphi}=T_{v \circ \varphi} T_{u \circ \varphi}$ on $A^{2}$ for every $\varphi \in \Phi$.

(c) $f \bar{k}-h \bar{g}$ is boundary n-harmonic.

Finally, only for $n \geq 3$, we show that the commuting property and the essential commuting property of Toeplitz operators with pluriharmonic symbols are equivalent. This will follow from Theorem 3.5 of Section 3 which asserts that the $n$-harmonicity and the boundary $n$-harmonicity are equivalent for functions of the form $f \bar{k}-h \bar{g}$ under consideration.

Theorem $1.4(n \geq 3)$. Let $u, v \in L^{\infty}$ be pluriharmonic symbols. Then the following statements are equivalent:

(a) $T_{u} T_{v}=T_{v} T_{u}$ on $A^{2}$.

(b) $T_{u}$ and $T_{v}$ are essentially commuting on $A^{2}$.

Remarks 1 . In case $n=1$, Theorem 1.1 together with Theorem 1.2 reduces to the result of Axler and C̆učković [1] mentioned before. To see this, consider the case $n=1$. Then, there are two cases: $I=I_{1}$ or $I=I_{0}$. If $I=I_{1}$, then $f_{0}, g_{0}, h_{0}$ and $k_{0}$ are constants. Now, since $v=\alpha_{1} u+\left(h_{0}+\bar{k}_{0}\right)-\alpha_{1}\left(f_{0}+\bar{g}_{0}\right)$ by (b2), we find 
that a nontrivial linear combination of $u$ and $v$ is constant. If $I=I_{0}$, then $p_{1}=0$ by (b1). Now, if condition (i) or (ii) holds, then (b2) shows that $u$ and $v$ are both antiholomorphic or both holomorphic, respectively. Similarly, (iii) or (iv) implies $u$ or $v$ is constant, respectively.

2. The class $\Phi$ has the property that if $u \in L^{\infty}$ is pluriharmonic and if $\varphi \in \Phi$, then $u \circ \varphi$ is also pluriharmonic. So, Theorem 1.1 as well as Theorem 1.2 can be applied to get more conditions equivalent to (b) of Theorem 1.3 .

3. Theorem 1.4 fails to hold for $n \leq 2$, as mentioned before. For a counterexample, see (3.5). Also, see an example at the end of Section 5.

This paper is arranged as follows. In Section 2 we collect basic materials which we need in later sections. In Section 3 we prove Theorem 1.2. Also, we show that the $n$-harmonicity and the boundary $n$-harmonicity of functions of certain forms are equivalent for $n \geq 3$. In Section 4 we prove Theorem 1.1. As an application we obtain a characterization of normal Toeplitz operators with pluriharmonic symbols. In Section 5 we prove Theorem 1.3. As a consequence we obtain Theorem 1.4 As an application we obtain a characterization of essentially normal Toeplitz operators with pluriharmonic symbols. As another application we recover the result of Sun and Zheng [14] mentioned above. In Section 6 we modify our arguments used in previous sections to obtain (essentially) semi-commuting Toeplitz operators with pluriharmonic symbols. It turns out that the semi-commuting property and the essential semi-commuting property are equivalent for $n \geq 2$.

\section{Preliminaries}

We collect several basic facts which we need in later sections.

Bergman projection. Since every point evaluation is a bounded linear functional on $A^{2}$, there corresponds to every $a \in D^{n}$ a unique function $K_{a} \in A^{2}$ which has the following reproducing property:

$$
f(a)=\left\langle f, K_{a}\right\rangle, \quad f \in A^{2},
$$

where the notation $\langle$,$\rangle denotes the inner product in L^{2}$ with respect to the measure $V$. The function $K_{a}$ is the well-known Bergman kernel and its explicit formula is given by

$$
K_{a}(z)=\prod_{j=1}^{n} \frac{1}{\left(1-\bar{a}_{j} z_{j}\right)^{2}}, \quad z, a \in D^{n} .
$$

The Bergman projection $P$ is the orthogonal projection from $L^{2}$ onto $A^{2}$. Thus, by the reproducing property (2.1), the projection $P$ can be represented by

$$
P \psi(a)=\int_{D^{n}} \psi \bar{K}_{a} d V, \quad a \in D^{n},
$$

for functions $\psi \in L^{2}$. It follows that $P$ naturally extends via the above formula to an integral operator from $L^{1}$ into $H\left(D^{n}\right)$. Moreover, we have $P f=f$ for functions $f \in A^{1}$. Here, $A^{p}=L^{p} \cap H\left(D^{n}\right)$. Also, it is well known that $P: L^{p} \rightarrow A^{p}$ is bounded for $p>1$. See, for example, Theorem 4.2.3 of [18] for details on the disk. The same proof works on $D^{n}$. 
Invariant mean value property. For each $a=\left(a_{1}, \ldots, a_{n}\right) \in D^{n}$, we let $\varphi_{a}(z)=$ $\left(\varphi_{a_{1}}\left(z_{1}\right), \cdots, \varphi_{a_{n}}\left(z_{n}\right)\right)$, where each $\varphi_{a_{i}}$ is the usual Möbius map on $D$ given by

$$
\varphi_{a_{i}}\left(z_{i}\right)=\frac{a_{i}-z_{i}}{1-\bar{a}_{i} z_{i}}, \quad z_{i} \in D
$$

Then $\varphi_{a} \in \operatorname{Aut}\left(D^{n}\right)$, the set of all automorphisms of $D^{n}$. Moreover, $\varphi_{a} \circ \varphi_{a}$ is the identity on $D^{n}$. Now, it is clear that if $u$ is $n$-harmonic, then so is $u \circ \varphi_{a}$ for each $a \in D^{n}$. Therefore, every $n$-harmonic function $u \in L^{1}$ satisfies the invariant mean value property

$$
\int_{D^{n}}\left(u \circ \varphi_{a}\right) d V=u(a), \quad a \in D^{n}
$$

However, the converse of the invariant mean value property is known to hold only for $n=1$. See 8$]$ and the references therein. Nevertheless, the converse turns out to be true in general with a certain additional hypothesis. To state it, we associate with each $u \in L^{1}$ its so-called radialization $\mathcal{R} u$ defined by

$$
\mathcal{R} u(z)=\int_{T^{n}} u\left(z_{1} \zeta_{1}, \ldots, z_{n} \zeta_{n}\right) d \sigma\left(\zeta_{1}, \ldots, \zeta_{n}\right)
$$

for $z=\left(z_{1}, \ldots, z_{n}\right) \in D^{n}$. Here and elsewhere, $T^{n}$ denotes the cartesian product of $n$ copies of the unit circle $T$ and $\sigma=\sigma_{n}$ is the normalized Haar measure on $T^{n}$. The following is taken from Corollary 3.7 of 8$]$.

Proposition 2.1. Let $u \in L^{1}$. Then $u$ is n-harmonic on $D^{n}$ if and only if

$$
\int_{D^{n}}\left(u \circ \varphi_{a}\right) d V=u(a)
$$

and $\mathcal{R}\left(u \circ \varphi_{a}\right) \in L^{\infty}$ for every $a \in D^{n}$.

Normalized kernel. We let $k_{a}$ denote the normalized kernel, namely,

$$
k_{a}=K_{a} \prod_{j=1}^{n}\left(1-\left|a_{j}\right|^{2}\right) .
$$

First, we mention that the set $\left\{k_{a}: a \in D^{n}\right\}$ spans a dense subset of $A^{2}$, because its orthogonal complement is $\{0\}$ by (2.1). Next, since the real Jacobian of $\varphi_{a}$ is given by $\left|k_{a}\right|^{2}$, we have a change-of-variable formula,

$$
\int_{D^{n}}\left(h \circ \varphi_{a}\right) d V=\int_{D^{n}} h\left|k_{a}\right|^{2} d V, \quad a \in D^{n},
$$

whenever the integrals make sense. In particular, we have by the mean value property

$$
\int_{D^{n}} f\left|k_{a}\right|^{2} d V=f(a), \quad a \in D^{n}
$$

for functions $f \in A^{1}$.

Hardy space. Given $p>0$, the Hardy space $H^{p}=H^{p}\left(D^{n}\right)$ is the space of all $f \in H\left(D^{n}\right)$ for which

$$
\|f\|_{H^{p}}^{p}=\sup _{0 \leq r<1} \int_{T^{n}}|f(r \zeta)|^{p} d \sigma(\zeta)<\infty .
$$

By an integration in polar coordinates using $n$-subharmonicity, we have $H^{p} \subset A^{p}$. 
It is well known that if $f \in H^{p}$, then $f(\zeta)=\lim _{r \rightarrow 1} f(r \zeta)$ exists at almost all points $\zeta \in T^{n}$. Moreover, we have $\log |f| \in L^{1}\left(T^{n}\right)$ for any nontrivial $f \in H^{p}$. In particular, if the boundary function of $f \in H^{p}$ vanishes on a set of positive measure in $T^{n}$, then $f$ itself must be identically 0 on $D^{n}$. See Theorem 3.4.2 of [11].

From the above definition, one can easily verify

$$
\mathcal{R}(f \bar{g}) \in L^{\infty}, \quad f, g \in H^{2} .
$$

Also, by using the $L^{p}$-boundedness of the Cauchy projection, one can easily verify the following.

Proposition 2.2. Let $f, g \in H\left(D^{n}\right)$ and assume $f+\bar{g} \in L^{\infty}$. Then we have $f, g \in H^{p}$ for all $p>0$.

Maximal ideal space. Let $\mathcal{M}$ be the maximal ideal space of $H^{\infty}$ which is defined to be the set of all multiplicative linear functionals on $H^{\infty}$. As is well known, the space $\mathcal{M}$ becomes a compact Hausdorff space as a subset of the dual of $H^{\infty}$ with weak-star topology. See Theorem 11.9 of [12] for details. Identifying $z \in D^{n}$ with the multiplicative evaluation functional $f \mapsto f(z)$, we can regard $D^{n}$ as a subset of $\mathcal{M}$.

Given $z \in D^{n}$, since $D^{n}$ is a subset of $\mathcal{M}$, we can think of $\varphi_{z}$ as a map from $D^{n}$ to $\mathcal{M}$. In other words, $\varphi_{z} \in \mathcal{M}^{D^{n}}$. Equipped with product topology, the function space $\mathcal{M}^{D^{n}}$ is compact by Tychonoff's theorem. Hence, for any net $\left\{\varphi_{z_{\alpha}}\right\}$ of automorphisms, there is a subnet $\left\{\varphi_{z_{\beta}}\right\}$ of $\left\{\varphi_{z_{\alpha}}\right\}$ such that $\varphi_{z_{\beta}}$ converges (pointwise) to a map $\varphi: D^{n} \rightarrow \mathcal{M}$. Now, we let

$$
\Phi=\operatorname{closure}\left\{\varphi_{z}: z \in D^{n}\right\} \backslash\left\{\varphi_{z}: z \in D^{n}\right\}
$$

where the closure is taken in $\mathcal{M}^{D^{n}}$.

We will use a couple of basic facts concerning the maximal ideal space $\mathcal{M}$ and the class $\Phi$. First, note that $H^{\infty} \subset C(\mathcal{M})$ via the Gelfand transform. For bounded pluriharmonic functions, we have the following.

Proposition 2.3. Each bounded pluriharmonic function on $D^{n}$ extends to a continuous function on $\mathcal{M}$.

In what follows we will use the same notation for a bounded pluriharmonic function and its continuous extension on $\mathcal{M}$.

Proposition 2.4. If a net $\left\{\varphi_{z_{\alpha}}\right\}$ of automorphisms converges to some $\varphi \in \Phi$, then for any pluriharmonic function $u \in L^{\infty}$, the function $u \circ \varphi_{z_{\alpha}}$ converges to $u \circ \varphi$ uniformly on every compact subset of $D^{n}$. So, $u \circ \varphi \in L^{\infty}$ is also pluriharmonic on $D^{n}$.

The above two propositions are proved in [16] on the ball. The same proofs work on the polydisk and thus proofs are omitted.

\section{3. $n$-HARMONIC FUNCTIONS}

In this section we prove Theorem 1.2, which will play an essential role in the proof of Theorem 1.1 We begin with a simple lemma.

Lemma 3.1. Suppose $J_{0}, J_{1}, J_{2}$ are pairwise disjoint subsets of $I$. Let $p_{j} \in$ $H\left(J_{0} \cup J_{j}\right) / H\left(J_{0}\right)$ for $j=1,2$ and assume $p_{1}+p_{2}=0$. Then $p_{1}=p_{2}=0$. 
Proof. Let $z^{j}=\left(z_{r}\right)_{r \in J_{j}}$ for $j=0,1,2$. Changing the coordinate system if necessary, we may write $p_{j}=p_{j}\left(z^{0}, z^{j}\right)$ for $j=1,2$. Now, taking $z^{2}=0$, we have $p_{1}\left(z^{0}, z^{1}\right)=-p_{2}\left(z^{0}, 0\right)$ and thus $p_{1} \in H\left(J_{0} \cup J_{1}\right) / H\left(J_{0}\right) \cap H\left(J_{0}\right)=\{0\}$. So, we have $p_{1}=p_{2}=0$. The proof is complete.

We are now ready to prove Theorem 1.2

Proof of Theorem 1.2. First suppose (a) and show (b). So, assume that the function $f \bar{k}-h \bar{g}$ is $n$-harmonic. Then, for each $r \in I$, we have $\partial_{r} \overline{\partial_{r}}(f \bar{k})=\partial_{r} \overline{\partial_{r}}(h \bar{g})$ and thus

$$
\left(\partial_{r} f\right)\left(\overline{\partial_{r} k}\right)=\left(\partial_{r} h\right)\left(\overline{\partial_{r} g}\right) .
$$

Let $I_{0}$ be the set of all $r \in I$ with $\left(\partial_{r} f\right)\left(\partial_{r} h\right)\left(\partial_{r} g\right)\left(\partial_{r} k\right)=0$. Then, for each $r \notin I_{0}$, we have

$$
\frac{\partial_{r} h}{\partial_{r} f}=\overline{\left(\frac{\partial_{r} k}{\partial_{r} g}\right)}
$$

and therefore there exists a constant $\beta_{r} \neq 0$ such that

$$
\partial_{r} h=\beta_{r} \partial_{r} f, \quad \partial_{r} k=\bar{\beta}_{r} \partial_{r} g .
$$

Now, define an equivalence relation on $I \backslash I_{0}$ by $r \sim s$ if and only if $\beta_{r}=\beta_{s}$ and let $I_{1}, \ldots, I_{m}$ be the equivalence classes induced by $\sim$. It follows that there are nonzero constants $\alpha_{1}, \ldots, \alpha_{m}$ such that

$$
\partial_{r} h=\alpha_{j} \partial_{r} f, \quad \partial_{r} k=\bar{\alpha}_{j} \partial_{r} g, \quad r \in I_{j},
$$

for each $j \geq 1$.

Note that, for $r \in I_{i}, s \in I_{j}$ with $i, j \geq 1$ and $i \neq j$, we have

$$
\left(\alpha_{i}-\alpha_{j}\right) \partial_{r} \partial_{s} f=\alpha_{i} \partial_{r} \partial_{s} f-\alpha_{j} \partial_{r} \partial_{s} f=\partial_{s} \partial_{r} h-\partial_{r} \partial_{s} h=0
$$

and thus $\partial_{r} \partial_{s} f=0$. This means that the power series of $f$ cannot contain any terms involving both $z_{r}$ and $z_{s}$ with $r \in I_{i}, s \in I_{j}$ whenever $i, j \geq 1$ and $i \neq j$. Similarly, the same is true for $g$. Also, since $\alpha_{j}$ 's are nonzero, the same holds for $h$ and $k$. Therefore, we may decompose functions $f, g, h$ and $k$ as

$$
f=\sum_{j=0}^{m} f_{j}, \quad g=\sum_{j=0}^{m} g_{j}, \quad h=\sum_{j=0}^{m} h_{j}, \quad k=\sum_{j=0}^{m} k_{j},
$$

where $f_{0}, g_{0}, h_{0}, k_{0} \in H\left(I_{0}\right)$ and $f_{j}, g_{j}, h_{j}, k_{j} \in H\left(I_{0} \cup I_{j}\right)$ for $j \geq 1$. Since we have $f_{0}, g_{0}, h_{0}, k_{0} \in H\left(I_{0}\right)$, we may further assume $f_{j}, g_{j}, h_{j}, k_{j} \in H\left(I_{0} \cup I_{j}\right) / H\left(I_{0}\right)$ for $j \geq 1$.

Now, we prove (b1) and (b2). Fix $j \geq 1$. Note that we have by (3.2)

$$
\partial_{r}\left(h_{j}-\alpha_{j} f_{j}\right)=\partial_{r}\left(h-\alpha_{j} f\right)=0
$$

for all $r \in I_{j}$. It follows that $h_{j}-\alpha_{j} f_{j} \in H\left(I_{0} \cup I_{j}\right) / H\left(I_{0}\right) \cap H\left(I_{0}\right)=\{0\}$ and thus $h_{j}=\alpha_{j} f_{j}$. Similarly, we have $k_{j}=\bar{\alpha}_{j} g_{j}$. Thus, (b1) and (b2) hold with $p_{j}=f_{j}$ and $q_{j}=g_{j}$ for $j \geq 1$.

Finally, we prove (b3). Let $r \in I_{0}$. Then by (3.1), one of the following four cases should occur:

$$
\begin{aligned}
& \text { (i) })^{\prime} \partial_{r} f=\partial_{r} h=0, \quad \text { (ii) } \partial_{r} k=\partial_{r} g=0 \text {, } \\
& \text { (iii) }{ }^{\prime} \partial_{r} f=\partial_{r} g=0, \quad \text { (iv) }{ }^{\prime} \partial_{r} k=\partial_{r} h=0 \text {. }
\end{aligned}
$$


In the case $(\mathrm{i})^{\prime}$, we have by (b2)

$$
\partial_{r} f_{0}+\sum_{j=1}^{m} \partial_{r} p_{j}=\partial_{r} h_{0}+\sum_{j=1}^{m} \alpha_{j} \partial_{r} p_{j}=0 .
$$

Note that, for each $j$, we have $\partial_{r} p_{j} \in H\left(I_{0} \cup I_{j}\right) / H\left(I_{0}\right)$, because $p_{j} \in H\left(I_{0} \cup I_{j}\right) /$ $H\left(I_{0}\right)$. Also, we have $\partial_{r} f_{0}, \partial_{r} h_{0} \in H\left(I_{0}\right)$. Thus, by repeated applications of Lemma 3.1, we conclude $\partial_{r} f_{0}=\partial_{r} h_{0}=0$ and $\partial_{r} p_{j}=0$ for each $j$, which is just the case (i). Similarly, the remaining cases (ii) ${ }^{\prime}$, (iii) ${ }^{\prime}$ and (iv) ${ }^{\prime}$ correspond to the cases (ii), (iii), and (iv), respectively. Therefore, we have (b).

Now, suppose (b) and show (c). By (b3), given $r \in I_{0}$, we have either $\partial_{r} f_{0}=$ $\partial_{r} p_{j}=0$ for all $j$ or $\partial_{r} k_{0}=\partial_{r} q_{j}=0$ for all $j$. This means that we can decompose $I_{0}=J_{1} \cup J_{2}$ where $J_{1} \cap J_{2}=\emptyset$ such that $f_{0} \in H\left(J_{1}\right), k_{0} \in H\left(J_{2}\right), p_{j} \in H\left(J_{1} \cup I_{j}\right)$, $q_{j} \in H\left(J_{2} \cup I_{j}\right)$ for all $j$. Since

$$
f \bar{k}-\sum_{j=1}^{m} \alpha_{j} p_{j} \bar{q}_{j}=f_{0} \bar{k}_{0}+\sum_{j=1}^{m} \alpha_{j} f_{0} \bar{q}_{j}+\sum_{j=1}^{m} p_{j} \bar{k}_{0}+\sum_{i \neq j} \alpha_{j} p_{i} \bar{q}_{j}
$$

by (b2), we see the function $f \bar{k}-\sum \alpha_{j} p_{j} \bar{q}_{j}$ can be written as a finite sum of functions of desired form. Also, a similar argument shows that the same is true for $h \bar{g}-\sum \alpha_{j} p_{j} \bar{q}_{j}$. Hence, we conclude (c).

Finally, it is trivial that (c) implies (a). The proof is complete.

As a special case of Theorem 1.2, we have the following consequence.

Corollary 3.2. Let $f, g \in H\left(D^{n}\right)$. Then the following statements are equivalent:

(a) $|f|^{2}-|g|^{2}$ is n-harmonic.

(b) There are pairwise disjoint sets $I_{1}, \ldots, I_{m}$ with $\bigcup_{j=1}^{m} I_{j}=I$ for some positive integer $m \leq n$, functions $p_{1}, \ldots, p_{m}$ with $p_{j} \in H\left(I_{j}\right)$ for each $j$, and unimodular constants $\alpha_{1}, \ldots, \alpha_{m}$ such that

$$
f=\sum_{j=1}^{m} p_{j}, \quad g=\sum_{j=1}^{m} \alpha_{j} p_{j}+\lambda
$$

for some constant $\lambda$.

Proof. By Theorem 1.2 with $h=g$ and $k=f$, we see that $|f|^{2}-|g|^{2}$ is $n$-harmonic if and only if there are pairwise disjoint sets $I_{0}, \ldots, I_{m}$ with $\bigcup_{j=0}^{m} I_{j}=I$ for some nonnegative integer $m \leq n$, functions $f_{0}, g_{0} \in H\left(I_{0}\right)$, constants $\alpha_{1}, \ldots, \alpha_{m}$, and $p_{1}, \ldots, p_{m}$ with $p_{j} \in H\left(I_{0} \cup I_{j}\right) / H\left(I_{0}\right)$ for each $j$, such that

$$
f=f_{0}+\sum_{j=1}^{m} p_{j}, \quad g=g_{0}+\sum_{j=1}^{m} \alpha_{j} p_{j}
$$

and

$$
\left|\alpha_{j}\right|^{2} p_{j}=p_{j}, \quad \partial_{r} f_{0}=\partial_{r} g_{0}=\partial_{r} p_{j}=0
$$

for all $r \in I_{0}$ and $j \geq 1$. By the first equation of the above, we may take $\left|\alpha_{j}\right|=1$. By the second equation, we see that functions $f_{0}, g_{0}$ are constant. Thus, we may take $f_{0}=0, I_{0}=\emptyset$ and $p_{j} \in H\left(I_{j}\right)$ for all $j \geq 1$. The proof is complete. 
Remark. In case $f, g, h, k \in H^{p}$ for some $p>0$, all the functions $f_{0}, g_{0}, h_{0}, k_{0}, p_{j}, q_{j}$ in (b) of Theorem 1.2 are also members of $H^{p}$. To see this, let $z^{j}=\left(z_{r}\right)_{r \in I_{j}}$ for $j=0, \ldots, m$. By changing the coordinate system if necessary, we may assume $z=\left(z^{0}, z^{1}, \ldots ., z^{m}\right)$ for $z \in D^{n}$. Also, write $f_{0}=f_{0}\left(z^{0}\right)$ and $p_{j}=p_{j}\left(z^{0}, z^{j}\right)$ for simplicity. Since the power series at the origin of each $p_{j}$ does not contain any nonzero terms in $H\left(I_{0}\right)$, one can easily verify $p_{j}\left(z^{0}, 0\right)=0$ for each $j$. Therefore, taking $z^{1}=\ldots=z^{m}=0$, we have $f\left(z^{0}, 0, \ldots, 0\right)=f_{0}\left(z^{0}\right)$. This yields $f_{0} \in H^{p}$ (see Lemma 3.3). Now, taking $z^{2}=\ldots=z^{m}=0$, we have $f\left(z^{0}, z^{1}, 0, \ldots, 0\right)-f_{0}\left(z^{0}\right)=$ $p_{1}\left(z^{0}, z^{1}\right)$ and therefore $p_{1} \in H^{p}$. Continuing this, we obtain $p_{j} \in H^{p}$ for all $j$. Similarly, we have $g_{0}, h_{0}, k_{0}, q_{j} \in H^{p}$ for all $j$. Also, by the proof of (b) $\Rightarrow$ (c) of Theorem 1.2, one can see that the functions $A_{i}, B_{i}$ in (c) of Theorem 1.2 can be chosen in the same class $H^{p}$.

For functions of the form $f \bar{k}-h \bar{g}$ with a certain regularity, the $n$-harmonicity and the boundary $n$-harmonicity turn out to be equivalent in the case $n \geq 3$, while they are different for $n=2$. In order to see this, we need the following lemma which might be known. A proof is included here for completeness.

Lemma 3.3. Let $m, \ell$ be integers with $1 \leq m<n, \ell \geq 0$ and assume $f \in H^{2}$. Then, $\partial_{1}^{\ell} f(z, \cdot) \in H^{2}\left(D^{n-m}\right)$ for each $z \in D^{m}$. Furthermore, there exists a set $E \subset T^{n-m}$ with $\sigma_{n-m}(E)=1$ with the following properties:

(a) $\partial_{1}^{\ell} f(z, \eta)=\lim _{r \rightarrow 1} \partial_{1}^{\ell} f(z, r \eta)$ exists for each $z \in D^{m}$ and $\eta \in E$.

(b) The function $\partial_{1}^{\ell} f(\cdot, \eta)$ is holomorphic on $D^{m}$ for each $\eta \in E$.

In the proof below we will use well-known facts about maximal functions. For a measurable function $\psi$ on $D^{m}$, let $N \psi$ be the nontangential maximal function of $\psi$ with respect to nontangential approach region of a fixed aperture. Also, given $u \in L^{1}\left(T^{n-m}\right)$, let $M u$ be the Hardy-Littlewood maximal function of $u$. As is well-known, the operator $M$ is bounded on $L^{2}\left(T^{n-m}\right)$. Also, it is well known that if $\psi$ is the Poisson integral of some $u \in L^{1}\left(T^{n-m}\right)$, then $N \psi \leq C M u$ for some constant $C$ independent of $u$.

Proof. Let $z=\left(z_{1}, \ldots, z_{m}\right) \in D^{m}$ and $\eta \in T^{n-m}$. Let $\max _{1 \leq j \leq m}\left|z_{j}\right|<t<1$. Then, for arbitrary $0<r<1$, we have by the Cauchy integral formula

$$
\partial_{1}^{\ell} f(z, r \eta)=\ell ! \int_{T^{m}} \frac{f(t \zeta, r \eta)}{\left(1-t^{-1} z_{1} \bar{\zeta}_{1}\right)^{\ell} \prod_{j=1}^{m}\left(1-t^{-1} z_{j} \bar{\zeta}_{j}\right)}\left(t^{-1} \bar{\zeta}_{1}\right)^{\ell} d \sigma_{m}(\zeta)
$$

and therefore

$$
\begin{aligned}
\int_{T^{n-m}} & \left|\partial_{1}^{\ell} f(z, r \eta)\right|^{2} d \sigma_{n-m}(\eta) \\
& \leq \frac{C_{\ell}}{\left(t-\left|z_{1}\right|\right)^{2 \ell} \prod_{j=1}^{m}\left(t-\left|z_{j}\right|\right)^{2}} \int_{T^{n-m}} \int_{T^{m}}|f(t \zeta, r \eta)|^{2} d \sigma_{m}(\zeta) d \sigma_{n-m}(\eta) \\
& \leq \frac{C_{\ell}}{\left(t-\left|z_{1}\right|\right)^{2 \ell} \prod_{j=1}^{m}\left(t-\left|z_{j}\right|\right)^{2}}\|f\|_{H^{2}}^{2}
\end{aligned}
$$

Hence, $\partial_{1}^{\ell} f(z, \cdot) \in H^{2}\left(D^{n-m}\right)$.

Now, pick a sequence of positive numbers $\left\{t_{j}\right\}$ increasing to 1 and let $K_{j}=\left\{t_{j} z\right.$ : $\left.z \in \bar{D}^{m}\right\}$ for $j \geq 1$. Fix $j$ and $t_{j}<t<1$. Then the above estimate shows

$$
\left|\partial_{1}^{\ell} f(z, r w)\right|^{2} \leq C_{j} \int_{T^{m}}|f(t \zeta, r w)|^{2} d \sigma_{m}(\zeta)
$$


for all $z \in K_{j}, 0<r \leq 1$ and $w \in D^{n-m}$. Here and in what follows, the letter $C_{j}=C_{j}(t)$ denotes various constants independent of $f, z, r$ and $w$. Let $z \in D^{m}$ and $0<r<1$. Then, it follows from (3.3) that

$$
\left(\sup _{z \in K_{j}} \mathcal{N} \partial_{1}^{\ell} f_{z, r}(\eta)\right)^{2} \leq C_{j} \int_{T^{m}} \mathcal{N} f_{t \zeta, r}(\eta)^{2} d \sigma_{m}(\zeta), \quad \eta \in T^{n-m},
$$

where we use the notation $h_{z, r}(w)=h(z, r w)$ for holomorphic functions $h$ on $D^{n}$. Integrating both sides of the above on $T^{n-m}$, we have

$$
\begin{aligned}
\int_{T^{n-m}} & \left(\sup _{z \in K_{j}} N \partial_{1}^{\ell} f_{z, r}(\eta)\right)^{2} d \sigma_{n-m}(\eta) \\
& \leq C_{j} \int_{T^{m}} \int_{T^{n-m}} N f_{t \zeta, r}(\eta)^{2} d \sigma_{n-m}(\eta) d \sigma_{m}(\zeta) \\
& \leq C_{j} \int_{T^{m}} \int_{T^{n-m}} M f_{t \zeta, r}(\eta)^{2} d \sigma_{n-m}(\eta) d \sigma_{m}(\zeta) \\
& \leq C_{j} \int_{T^{m}} \int_{T^{n-m}}\left|f_{t \zeta, r}(\eta)\right|^{2} d \sigma_{n-m}(\eta) d \sigma_{m}(\zeta) \\
& =C_{j} \int_{T^{m}} \int_{T^{n-m}}|f(t \zeta, r \eta)|^{2} d \sigma_{n-m}(\eta) d \sigma_{m}(\zeta) \\
& \leq C_{j}\|f\|_{H^{2}}^{2} .
\end{aligned}
$$

Thus, by Fatou's lemma, we have

$$
\int_{T^{n-m}}\left(\sup _{z \in K_{j}} \mathcal{N} \partial_{1}^{\ell} f_{z, 1}(\eta)\right)^{2} d \sigma_{n-m}(\eta) \leq C_{j}\|f\|_{H^{2}}^{2}
$$

Having the above inequality, one may now follow the well-known proof of Fatou's theorem to conclude that there exists a set $E_{j} \subset T^{n-m}$ with $\sigma_{n-m}\left(E_{j}\right)=1$ such that nontangential limits of $\partial_{1}^{\ell} f(z, \cdot)$ exist at all points in $E_{j}$ for each $z \in K_{j}$. Let $E=\bigcap_{j=1}^{\infty} E_{j}$. Then we still have $\sigma_{n-m}(E)=1$ and nontangential limits of $\partial_{1}^{\ell} f(z, \cdot)$ exist at all points in $E$ for each $z \in D^{m}$. This proves (a).

Note that (3.4) yields

$$
\sup _{z \in K_{j}} N \partial_{1}^{\ell} f_{z, 1}(\eta)<\infty
$$

for almost all points $\eta$ in $T^{n-m}$. We may assume the above holds for $\eta \in E_{j}$. Thus, given a compact set $K \subset D^{m}$, we have

$$
\sup _{z \in K} N \partial_{1}^{\ell} f_{z, 1}(\eta)<\infty
$$

for each $\eta \in E$. In particular, given $\eta \in E$, we see that functions $\partial_{1}^{\ell} f(\cdot, r \eta)$, $0<r<1$, form a normal family and thus (b) holds. The proof is complete.

The following is taken from Lemma 9 of [3].

Lemma 3.4. Let $\Omega$ be a given connected open subset of $\mathbb{C}^{n}$. If $A_{i}$ and $B_{i}(1 \leq i \leq \ell)$ are holomorphic functions such that $\sum_{i=1}^{\ell} A_{i} \bar{B}_{i}=0$ on $\Omega$, then $\sum_{i=1}^{\ell} A_{i}(z) \bar{B}_{i}(w)=$ 0 for all $z, w \in \Omega$. 
Now, we prove the following theorem, which does not extend to $n \leq 2$. For $n=1$, it is not hard to find counterexamples. For $n=2$, we have a counterexample:

$$
f=-g=\left(1-z_{1}\right)\left(1-z_{2}\right), \quad h=k=\left(1+z_{1}\right)\left(1+z_{2}\right) .
$$

Theorem $3.5(n \geq 3)$. Let $f, h, k, g \in H^{2}$. Then the following statements are equivalent:

(a) $f \bar{k}-h \bar{g}$ is n-harmonic.

(b) $f \bar{k}-h \bar{g}$ is boundary $n$-harmonic.

Proof. The implication (a) $\Rightarrow$ (b) is trivial. We prove (b) $\Rightarrow($ a). So, assume (b). By symmetry we only need to prove

$$
\left(\partial_{1} f\right)\left(\overline{\partial_{1} k}\right)=\left(\partial_{1} h\right)\left(\overline{\partial_{1} g}\right) .
$$

First, let us introduce some notation. For simplicity, put $F=\partial_{1} f, G=\partial_{1} g$, $H=\partial_{1} h$ and $K=\partial_{1} k$. Then, by Lemma 3.3, there exists a set $E \subset T$ with $\sigma_{1}(E)=1$ such that, given $\eta \in E$, the functions $F(\cdot, \eta), G(\cdot, \eta), H(\cdot, \eta)$ and $K(\cdot, \eta)$ are holomorphic on $D^{n-1}$. Also, we may assume that, given $\zeta \in E$, the functions $F(\cdot, \zeta, \cdot), G(\cdot, \zeta, \cdot), H(\cdot, \zeta, \cdot)$ and $K(\cdot, \zeta, \cdot)$ are holomorphic on $D^{n-1}=D^{n-2} \times D$.

Now, since we have

$$
\lim _{a \rightarrow \partial D^{n}} \widetilde{\Delta}_{1}(f \bar{k}-h \bar{g})(a)=0
$$

by assumption, it follows that

$$
\lim _{t \rightarrow 1}(F \bar{K}-H \bar{G})(z, t \eta)=0
$$

for all $z \in D^{n-1}$ and $\eta \in T$. In particular, we obtain

$$
(F \bar{K}-H \bar{G})(z, \eta)=0
$$

for all $z \in D^{n-1}$ and $\eta \in E$. Thus, we have by Lemma 3.4

$$
F(z, \eta) \overline{K(w, \eta)}=H(z, \eta) \overline{G(w, \eta)}
$$

for all $z, w \in D^{n-1}$ and $\eta \in E$.

Let $E_{f}$ be the set of all $\eta \in E$ such that $F(\cdot, \eta)=0$. Define the sets $E_{g}, E_{h}$ and $E_{k}$ in a similar way. First, consider the case where one of the sets $E_{f}, E_{g}, E_{h}$ and $E_{k}$ is of positive $\sigma_{1}$-measure. Without loss of generality, assume $\sigma_{1}\left(E_{f}\right)>0$. Let $z \in D^{n-1}$. Note that $F(z, \cdot) \in H^{2}(D)$ by Lemma 3.3 . Since the boundary function of $F(z, \cdot)$ vanishes on a set of positive $\sigma_{1}$-measure, we have $F(z, \cdot)=0$ on $D$. It follows that $F=0$ on $D^{n}$. Thus, we see from (3.7) that

$$
H(z, \eta) G(z, \eta)=0
$$

for all $z \in D^{n-1}$ and $\eta \in E$. This means that, for each $z \in D^{n-1}$, the boundary function of $H(z, \cdot) G(z, \cdot) \in H^{1}(D)$ vanishes on $E$. Hence, $H G=0$ on $D^{n}$. So, we have (3.6).

Now, assume that all the sets $E_{f}, E_{g}, E_{h}$ and $E_{k}$ are of $\sigma_{1}$-measure 0 . We may further assume that, for each $\eta \in E$, all the functions $F(\cdot, \eta), G(\cdot, \eta), H(\cdot, \eta)$ and $K(\cdot, \eta)$ are not identically 0 on $D^{n-1}$. Thus, we see from (3.7) that, for each $\eta \in E$, there exists a constant $\alpha(\eta)$ such that

$$
F(b, \lambda, \eta)=\alpha(\eta) H(b, \lambda, \eta)
$$

for all $b \in D^{n-2}$ and $\lambda \in D$. 
Repeating exactly the same argument as above, we may assume that, given $\zeta \in E$, the functions $F(\cdot, \zeta, \cdot), H(\cdot, \zeta, \cdot)$ are not identically 0 on $D^{n-1}$ and there is a constant $\beta(\zeta)$ such that

$$
F(b, \zeta, \lambda)=\beta(\zeta) H(b, \zeta, \lambda)
$$

for all $b \in D^{n-2}$ and $\lambda \in D$.

Now, choose $b_{0} \in D^{n-2}$ for which $F\left(b_{0}, \cdot\right)$ and $H\left(b_{0}, \cdot\right)$ are not identically 0 . By Lemma 3.3, we have $F\left(b_{0}, \cdot\right), H\left(b_{0}, \cdot\right) \in H^{2}\left(D^{2}\right)$ (it is in this step where we use the hypothesis $n \geq 3)$. Hence, $F\left(b_{0}, \cdot\right)$ and $H\left(b_{0}, \cdot\right)$ have nonzero boundary values at almost all points of $T^{2}$. Therefore, we may further assume that $F\left(b_{0}, \cdot\right)$ and $H\left(b_{0}, \cdot\right)$ have nonzero boundary values on $E \times E$. Thus, given $\eta, \zeta \in E$, we obtain $\alpha(\eta)=\beta(\zeta)$ by (3.8) and (3.9). It follows that $\alpha(\eta)=\alpha$ is also independent of $\eta$. We now have

$$
F(z, \eta)=\alpha H(z, \eta)
$$

for all $z \in D^{n-1}$ and $\eta \in E$. This yields $F=\alpha H$ on $D^{n}$ as before. Similarly, we have $G=\bar{\alpha} K$. So, (3.6) holds. The proof is complete.

Now, Theorem 1.4 follows from Theorem [1.1, Theorem 1.3 and Theorem 3.5 Also, as a corollary of the proof of Theorem [3.5, we have the following.

Corollary $3.6(n \geq 2)$. Let $f, g \in H^{2}$. Then the following statements are equivalent:

(a) $f \bar{g}$ is n-harmonic.

(b) $f \bar{g}$ is boundary n-harmonic.

\section{Commuting Toeplitz operators}

In this section we prove Theorem 1.1. The following fact is very useful for our purpose.

Proposition 4.1. Let $f, g \in A^{2}$. If $\partial_{j} f=0$ or $\partial_{j} g=0$ for each $j$, then we have

$$
P\left(f \bar{g} K_{a}\right)=f \overline{g(a)} K_{a}
$$

for $a \in D^{n}$. The converse also holds for $f, g \in H^{2}$.

Proof. Suppose $\partial_{j} f=0$ or $\partial_{j} g=0$ for each $j$. Then there are disjoint sets $J_{1}, J_{2}$ with $J_{1} \cup J_{2}=I$ such that $f \in H\left(J_{1}\right)$ and $g \in H\left(J_{2}\right)$. Write $z^{j}=\left(z_{r}\right)_{r \in J_{j}}$ for $j=1,2$. By changing the coordinate system if needed, we may write $z=\left(z^{1}, z^{2}\right)$ for $z \in D^{n}$. By assumption, we may regard $f$ and $g$ as functions holomorphic on lower-dimensional polydisks. That is, we may write $f(z)=f\left(z^{1}\right)$ and $g(z)=g\left(z^{2}\right)$ for $z \in D^{n}$. Also, note $K_{a}(z)=K_{a^{1}}\left(z^{1}\right) K_{a^{2}}\left(z^{2}\right)$ for $z, a \in D^{n}$. Here, we abuse the notation $K_{a^{1}}$ and $K_{a^{2}}$ for the kernel functions on the corresponding lower dimensional polydisks. Thus, for every $a, z \in D^{n}$, we have

$$
\begin{aligned}
\left\langle f K_{a}, g K_{z}\right\rangle & =\left\langle f K_{a^{1}} K_{a^{2}}, g K_{z^{1}} K_{z^{2}}\right\rangle \\
& =\left\langle f K_{a^{1}}, K_{z^{1}}\right\rangle\left\langle K_{a^{2}}, g K_{z^{2}}\right\rangle \\
& =\left\langle f K_{a^{1}}, K_{z^{1}}\right\rangle \overline{\left\langle g K_{z^{2}}, K_{a^{2}}\right\rangle} \\
& =f\left(z^{1}\right) K_{a^{1}}\left(z^{1}\right) \overline{g\left(a^{2}\right)} \overline{K_{z^{2}}\left(a^{2}\right)} \\
& =f(z) \overline{g(a)} K_{a}(z)
\end{aligned}
$$


and therefore

$$
P\left(f \bar{g} K_{a}\right)(z)=\left\langle f \bar{g} K_{a}, K_{z}\right\rangle=\left\langle f K_{a}, g K_{z}\right\rangle=f(z) \overline{g(a)} K_{a}(z)
$$

for every $a, z \in D^{n}$.

Now, let $f, g \in H^{2}$ and assume (4.1) holds. Let $a \in D^{n}$ be an arbitrary point. Then, by (2.3) we have

$$
\left\langle P\left(f \bar{g} k_{a}\right), k_{a}\right\rangle=\left\langle f \overline{g(a)} k_{a}, k_{a}\right\rangle=f(a) \overline{g(a)} .
$$

On the other hand, we have

$$
\left\langle P\left(f \bar{g} k_{a}\right), k_{a}\right\rangle=\left\langle f \bar{g} k_{a}, k_{a}\right\rangle=\int_{D^{n}}(f \bar{g}) \circ \varphi_{a} d V
$$

by 2.2. It follows that

$$
\int_{D^{n}}(f \bar{g}) \circ \varphi_{a} d V=f(a) \overline{g(a)}
$$

Note $\mathcal{R}(f \bar{k}) \in L^{\infty}$ by (2.4). Now, by Proposition [2.1] we conclude that $f \bar{g}$ is $n$-harmonic and therefore $\partial_{j} f=0$ or $\partial_{j} g=0$ for each $j$. This completes the proof.

We now turn to the proof of Theorem 1.1.

Proof of Theorem 1.1. We first prove (a) $\Rightarrow(\mathrm{b})$. So, assume $T_{u} T_{v}=T_{v} T_{u}$ on $A^{2}$. Since $u$ and $v$ are bounded, the functions $f, g, h$ and $k$ are all in $H^{2}$ and hence in $A^{2}$ by Proposition 2.2. Fix a point $a \in D^{n}$. By Proposition 4.1, we have $P\left(\bar{g} k_{a}\right)=\overline{g(a)} k_{a}$ and hence

$$
T_{f+\bar{g}} k_{a}=P\left[(f+\bar{g}) k_{a}\right]=[f+\overline{g(a)}] k_{a} .
$$

Therefore, we have

$$
\begin{aligned}
T_{h+\bar{k}} T_{f+\bar{g}} k_{a} & =P\left[(h+\bar{k})(f+\overline{g(a)}) k_{a}\right] \\
& =f h k_{a}+h \overline{g(a)} k_{a}+\overline{g(a)} \overline{k(a)} k_{a}+P\left(f \bar{k} k_{a}\right) .
\end{aligned}
$$

Similarly, we also have

$$
T_{f+\bar{g}} T_{h+\bar{k}} k_{a}=f h k_{a}+f \overline{k(a)} k_{a}+\overline{g(a)} \overline{k(a)} k_{a}+P\left(h \bar{g} k_{a}\right) .
$$

It follows that

$$
\left[T_{f+\bar{g}} T_{h+\bar{k}}-T_{h+\bar{k}} T_{f+\bar{g}}\right] k_{a}=[f \overline{k(a)}-h \overline{g(a)}] k_{a}-P\left[(f \bar{k}-h \bar{g}) k_{a}\right] .
$$

Since $T_{f+\bar{g}} T_{h+\bar{k}}=T_{h+\bar{k}} T_{f+\bar{g}}$ by assumption, we get

$$
P\left[(f \bar{k}-h \bar{g}) k_{a}\right]=[f \overline{k(a)}-h \overline{g(a)}] k_{a} .
$$

Now, as in the proof of the second part of Proposition 4.1 the above leads to the $n$-harmonicity of $f \bar{k}-h \bar{g}$.

Next, we prove (b) $\Rightarrow(\mathrm{a})$. So, assume $f \bar{k}-h \bar{g}$ is $n$-harmonic. Note that the set $\left\{k_{a}: a \in D^{n}\right\}$ spans a dense subset of $A^{2}$. Thus, in order to prove $T_{u} T_{v}=T_{v} T_{u}$, it is sufficient to show

$$
P\left[(f \bar{k}-h \bar{g}) k_{a}\right]=[f \overline{k(a)}-h \overline{g(a)}] k_{a}, \quad a \in D^{n},
$$

by (4.2). 
Now, write $f \bar{k}-h \bar{g}=\sum A_{i} \bar{B}_{i}$ where $A_{i}, B_{i}$ are functions as in (c) of Theorem 1.2. Let $z, a \in D^{n}$. Then, by Lemma 3.4 we have

$$
f(z) \overline{k(a)}-h(z) \overline{g(a)}=\sum A_{i}(z) \overline{B_{i}(a)} .
$$

By the remark at the end of Section 3, we may assume $A_{i}, B_{i} \in A^{2}$. It follows from Proposition 4.1 that

$$
\begin{aligned}
P\left[(f \bar{k}-h \bar{g}) k_{a}\right](z) & =\sum P\left(A_{i} \bar{B}_{i} k_{a}\right)(z) \\
& =\sum A_{i}(z) \overline{B_{i}(a)} k_{a}(z) \\
& =[f(z) \overline{k(a)}-h(z) \overline{g(a)}] k_{a}(z) .
\end{aligned}
$$

So, we conclude (4.3), as desired. The proof is complete.

Note that the adjoint of $T_{u}$ is $T_{\bar{u}}$. It follows that $T_{u}$ is normal if and only if $T_{u} T_{\bar{u}}=T_{\bar{u}} T_{u}$. Thus, by Theorem 1.1 and Corollary [3.2, we have the following.

Corollary 4.2. Let $u \in L^{\infty}$ be a pluriharmonic symbol. Then the following statements are equivalent:

(a) $T_{u}$ is normal on $A^{2}$.

(b) There are pairwise disjoint sets $I_{1}, \ldots, I_{m}$ with $\bigcup_{j=1}^{m} I_{j}=I$ for some positive integer $m \leq n$, functions $p_{1}, \ldots, p_{m}$ with $p_{j} \in H\left(I_{j}\right)$ for each $j$, and unimodular constants $\alpha_{1}, \ldots, \alpha_{m}$ such that

$$
u=\sum_{j=1}^{m} \alpha_{j}\left(p_{j}+\bar{p}_{j}\right)+\lambda
$$

for some constant $\lambda$.

Remarks 1 . In the case $n=1$, (4.4) is equivalent to the fact that $u(D)$ is contained in a straight line, which is also pointed out in 1]. However, such a characterization for a normal Toeplitz operator is no longer true for $n>1$. In fact, it is not hard to see that $u\left(D^{n}\right)$ is contained in a straight line if and only if $u=\alpha(p+\bar{p})+\lambda$ for some constants $\alpha, \lambda$ and $p \in H\left(D^{n}\right)$, which belongs to only a small part of functions of the form (4.4).

2. The same arguments used in this section work for the Toeplitz operators on the Hardy space $H^{2}$. Thus, the Hardy space versions of Theorem [.1]and Corollary 4.2 are also valid.

\section{Essentially commuting Toeplitz operators}

In this section we prove Theorem 1.3. The proof will be completed by proving the following sequence of implications:

$$
(\mathrm{a}) \Rightarrow(\mathrm{b}) \Rightarrow(\mathrm{c}) \Rightarrow(\mathrm{b}) \Rightarrow(\mathrm{a}) \text {. }
$$

Since proofs are somewhat long, we will prove each implication separately.

For the proof of the implication (a) $\Rightarrow(\mathrm{b})$, we introduce some notation. For each $a \in D^{n}$, we define a linear operator $U_{a}$ on $L^{2}$ by

$$
U_{a} \psi=\left(\psi \circ \varphi_{a}\right) k_{a}
$$


for $\psi \in L^{2}$. One can readily see that $U_{a}$ is an isometry taking $A^{2}$ onto itself. Also, since $\varphi_{a} \circ \varphi_{a}$ is the identity on $D^{n}$, one can see that $U_{a} U_{a}$ is the identity. Moreover, for $u \in L^{\infty}$, we have

$$
T_{u \circ \varphi_{a}}=U_{a} T_{u} U_{a}, \quad a \in D^{n} .
$$

This is proved in [1] on $D$ and the same proof works on $D^{n}$.

Proof of (a) $\Rightarrow(\mathrm{b})$. Let $\varphi \in \Phi$. Since the set $\left\{k_{a}: a \in D^{n}\right\}$ spans a dense subset of $A^{2}$, it is sufficient to show that

$$
\left(T_{u \circ \varphi} T_{v \circ \varphi}-T_{v \circ \varphi} T_{u \circ \varphi}\right) k_{a}=0, \quad a \in D^{n} .
$$

Choose a net $\left\{w_{\alpha}\right\}$ in $D^{n}$ such that $\varphi_{w_{\alpha}} \rightarrow \varphi$. First, note $u \circ \varphi_{w_{\alpha}} \rightarrow u \circ \varphi$ and $v \circ \varphi_{w_{\alpha}} \rightarrow v \circ \varphi$ uniformly on every compact subset of $D^{n}$ by Proposition 2.4 Moreover, since $u$ and $v$ are bounded, we have

$$
u \circ \varphi_{w_{\alpha}} \rightarrow u \circ \varphi, \quad v \circ \varphi_{w_{\alpha}} \rightarrow v \circ \varphi \quad \text { in } L^{2} .
$$

Fix $a \in D^{n}$. Then it follows from the above that $\left(v \circ \varphi_{w_{\alpha}}\right) k_{a} \rightarrow(v \circ \varphi) k_{a}$ in $L^{2}$. So, $P\left[\left(v \circ \varphi_{w_{\alpha}}\right) k_{a}\right] \rightarrow P\left[(v \circ \varphi) k_{a}\right]$ in $L^{2}$. Since $u \circ \varphi_{w_{\alpha}}$ is bounded and converges pointwise to $u \circ \varphi$, it is not hard to see

$$
P\left[u \circ \varphi_{w_{\alpha}} P\left(v \circ \varphi_{w_{\alpha}} k_{a}\right)\right] \rightarrow P\left[u \circ \varphi P\left(v \circ \varphi k_{a}\right)\right] \text { in } L^{2} .
$$

In other words,

$$
T_{u \circ \varphi_{w_{\alpha}}} T_{v \circ \varphi_{w_{\alpha}}} k_{a} \rightarrow T_{u \circ \varphi} T_{v \circ \varphi} k_{a} \quad \text { in } \quad L^{2} .
$$

Similarly, we have

$$
T_{v \circ \varphi_{w_{\alpha}}} T_{u \circ \varphi_{w_{\alpha}}} k_{a} \rightarrow T_{v \circ \varphi} T_{u \circ \varphi} k_{a} \quad \text { in } L^{2} .
$$

It follows from (5.1) that

$$
\begin{aligned}
\left\|\left(T_{u \circ \varphi} T_{v \circ \varphi}-T_{v \circ \varphi} T_{u \circ \varphi}\right) k_{a}\right\|_{2} & =\lim _{\alpha}\left\|\left(T_{u \circ \varphi_{w_{\alpha}}} T_{v \circ \varphi_{w_{\alpha}}}-T_{v \circ \varphi_{w_{\alpha}}} T_{u \circ \varphi_{w_{\alpha}}}\right) k_{a}\right\|_{2} \\
& =\lim _{\alpha}\left\|U_{w_{\alpha}}\left(T_{u} T_{v}-T_{v} T_{u}\right) U_{w_{\alpha}} k_{a}\right\|_{2} \\
& =\lim _{\alpha}\left\|\left(T_{u} T_{v}-T_{v} T_{u}\right) U_{w_{\alpha}} k_{a}\right\|_{2},
\end{aligned}
$$

where \|\|$_{2}$ denotes the $L^{2}$-norm. It is easy to see that $U_{w_{\alpha}} k_{a}$ converges to 0 weakly in $A^{2}$. Hence, the compactness of $T_{u} T_{v}-T_{v} T_{u}$ yields (5.2). This completes the proof.

For the proof of equivalence $(\mathrm{b}) \Leftrightarrow(\mathrm{c})$, we first prove the following lemma. In the proof below, we will use the well-known fact that

$$
\widetilde{\Delta}_{j}(u \circ \varphi)=\left(\widetilde{\Delta}_{j} u\right) \circ \varphi, \quad j=1, \ldots, n,
$$

for all $\varphi \in \operatorname{Aut}\left(D^{n}\right)$ and $u \in C^{2}\left(D^{n}\right)$.

Lemma 5.1. Let $u=f+\bar{g}, v=h+\bar{k}$ be as in the hypothesis of Theorem 1.3.

Suppose $\left\{\varphi_{w_{\alpha}}\right\}$ is a net such that $\varphi_{w_{\alpha}} \rightarrow \varphi \in \Phi$. If $u \circ \varphi=F+\bar{G}, v \circ \varphi=H+\bar{K}$ where $F, G, H, K \in H\left(D^{n}\right)$, then

$$
\widetilde{\Delta}_{j}(f \bar{k}-h \bar{g}) \circ \varphi_{w_{\alpha}} \rightarrow \widetilde{\Delta}_{j}(F \bar{K}-H \bar{G})
$$

for each $j$. 
Proof. Fix $j$. Put $f_{\alpha}=f \circ \varphi_{w_{\alpha}}-f\left(w_{\alpha}\right)$ and $k_{\alpha}=k \circ \varphi_{w_{\alpha}}-k\left(w_{\alpha}\right)$ for simplicity. First, by (5.3), we have

$$
\widetilde{\Delta}_{j}\left(f_{\alpha} \bar{k}_{\alpha}-h_{\alpha} \bar{g}_{\alpha}\right)=\widetilde{\Delta}_{j}\left[(f \bar{k}-h \bar{g}) \circ \varphi_{w_{\alpha}}\right]=\widetilde{\Delta}_{j}(f \bar{k}-h \bar{g}) \circ \varphi_{w_{\alpha}} .
$$

Thus, it remains to show

$$
\widetilde{\Delta}_{j}\left(f_{\alpha} \bar{k}_{\alpha}-h_{\alpha} \bar{g}_{\alpha}\right) \rightarrow \widetilde{\Delta}_{j}(F \bar{K}-H \bar{G}) .
$$

Note that

$$
u \circ \varphi_{w_{\alpha}}-u\left(w_{\alpha}\right) \rightarrow u \circ \varphi-u \circ \varphi(0)
$$

uniformly on every compact subset of $D^{n}$. In particular, since $u$ and $v$ are bounded,

$$
u \circ \varphi_{w_{\alpha}}-u\left(w_{\alpha}\right) \rightarrow u \circ \varphi-u \circ \varphi(0) \text { in } L^{2} .
$$

Now, using the $L^{2}$-boundedness of the Bergman projection $P$, we have

$$
P\left[u \circ \varphi_{w_{\alpha}}-u\left(w_{\alpha}\right)\right] \rightarrow P[u \circ \varphi-u \circ \varphi(0)] \text { in } L^{2} .
$$

Note that an application of Proposition 4.1 yields

$$
P\left[u \circ \varphi_{w_{\alpha}}-u\left(w_{\alpha}\right)\right]=f_{\alpha}, \quad P[u \circ \varphi-u \circ \varphi(0)]=F-F(0) .
$$

Hence, $f_{\alpha} \rightarrow F-F(0)$ in $L^{2}$. It follows that $f_{\alpha} \rightarrow F-F(0)$ uniformly on every compact subset of $D^{n}$ and therefore $\partial_{j} f_{\alpha} \rightarrow \partial_{j} F$. Applying the same reasoning to $\bar{v}$, we have $\partial_{j} k_{\alpha} \rightarrow \partial_{j} K$. Since

$$
\widetilde{\Delta}_{j}\left(f_{\alpha} \bar{k}_{\alpha}\right)(z)=\left(1-\left|z_{j}\right|^{2}\right)^{2} \partial_{j} f_{\alpha} \overline{\partial_{j} k_{\alpha}},
$$

it follows that

$$
\widetilde{\Delta}_{j}\left(f_{\alpha} \bar{k}_{\alpha}\right) \rightarrow\left(1-\left|z_{j}\right|^{2}\right)^{2} \partial_{j} F \overline{\partial_{j} K}=\widetilde{\Delta}_{j}(F \bar{K}) .
$$

Similarly, we have $\widetilde{\Delta}_{j}\left(h_{\alpha} \bar{g}_{\alpha}\right) \rightarrow \widetilde{\Delta}_{j}(H \bar{G})$. Hence, (5.4) holds. This completes the proof.

We now prove that (b) implies (c) and vice versa.

Proof of $(\mathrm{b}) \Rightarrow(\mathrm{c})$. It is sufficient to show that, for a given net $\left\{w_{\alpha}\right\}$ such that $\varphi_{w_{\alpha}} \rightarrow \varphi$ for some $\varphi \in \Phi$,

$$
\widetilde{\Delta}_{j}(f \bar{k}-h \bar{g})\left(w_{\alpha}\right) \rightarrow 0
$$

holds for each $j$. So, fix a net $\left\{w_{\alpha}\right\}$ such that $\varphi_{w_{\alpha}} \rightarrow \varphi$ for some $\varphi \in \Phi$ and let $F, G, H, K$ be as in Lemma 5.1. Since $T_{u \circ \varphi}$ and $T_{v \circ \varphi}$ commute by assumption, Theorem 1.1 shows the function $F \bar{K}-H \bar{G}$ is $n$-harmonic. Hence $\widetilde{\Delta}_{j}(F \bar{K}-H \bar{G})=0$ for each $j$. Consequently, by Lemma 5.1 with evaluation at the origin, we have (5.5) as desired. The proof is complete.

Proof of (c) $\Rightarrow$ (b). Let $\varphi \in \Phi$ and assume $\varphi_{w_{\alpha}} \rightarrow \varphi$. Let $u \circ \varphi=F+\bar{G}$ and $v \circ \varphi=H+\bar{K}$ as before. Fix an arbitrary point $a \in D^{n}$ and put $z_{\alpha}=\varphi_{w_{\alpha}}(a)$. Since $\varphi_{a} \circ \varphi_{w_{\alpha}} \circ \varphi_{z_{\alpha}} \in \operatorname{Aut}\left(D^{n}\right)$ fixes the origin, it is a unitary transformation, say $U_{a, \alpha}$. Thus we have

$$
\varphi_{z_{\alpha}}=\varphi_{w_{\alpha}} \circ \varphi_{a} \circ U_{a, \alpha} .
$$

Since the set of all unitary transformations is compact, we may assume $U_{a, \alpha}$ converges to some unitary transformation $U_{a}$. Now, for a given function $\psi \in H^{\infty}$, since $\psi \circ \varphi_{w_{\alpha}} \rightarrow \psi \circ \varphi$ uniformly on every compact subset of $D^{n}$ and $\varphi_{a} \circ U_{a, \alpha} \rightarrow \varphi_{a} \circ U_{a}$, we see that $\psi \circ \varphi_{w_{\alpha}} \circ \varphi_{a} \circ U_{a, \alpha} \rightarrow \psi \circ \varphi \circ \varphi_{a} \circ U_{a}$. This, together with (5.6), shows $\varphi_{z_{\alpha}} \rightarrow \tilde{\varphi}$ where $\tilde{\varphi}=\varphi \circ \varphi_{a} \circ U_{a}$. By the same argument, we have $u \circ \varphi_{z_{\alpha}} \rightarrow u \circ \tilde{\varphi}$ 
and $v \circ \varphi_{z_{\alpha}} \rightarrow v \circ \tilde{\varphi}$ uniformly on every compact subset of $D^{n}$. Note that $\varphi \in \Phi$ implies $w_{\alpha} \rightarrow \partial D^{n}$ and thus $z_{\alpha} \rightarrow \partial D^{n}$. So, $\tilde{\varphi} \in \Phi$.

Now, since $u \circ \tilde{\varphi}=F \circ \varphi_{a} \circ U_{a}+\overline{G \circ \varphi_{a} \circ U_{a}}$ and $v \circ \tilde{\varphi}=H \circ \varphi_{a} \circ U_{a}+\overline{K \circ \varphi_{a} \circ U_{a}}$, it follows from Lemma 5.1 and $(5.3)$ that

$$
\begin{aligned}
0 & =\lim _{\alpha} \widetilde{\Delta}_{j}[f \bar{k}-h \bar{g}]\left(z_{\alpha}\right) \\
& =\widetilde{\Delta}_{j}\left[(F \bar{K}-H \bar{G}) \circ \varphi_{a} \circ U_{a}\right](0) \\
& =\widetilde{\Delta}_{j}[F \bar{K}-H \bar{G}]\left(\varphi_{a} \circ U_{a}(0)\right) \\
& =\widetilde{\Delta}_{j}[F \bar{K}-H \bar{G}](a)
\end{aligned}
$$

for all $j$. So, the function $F \bar{K}-H \bar{G}$ is $n$-harmonic. Thus, $T_{u \circ \varphi}$ and $T_{v \circ \varphi}$ commute by Theorem 1.1. The proof is complete.

For the proof of the implication (b) $\Rightarrow(a)$, we introduce some notation. Given a pair of bounded pluriharmonic symbols $u=f+\bar{g}$ and $v=h+\bar{k}$ where $f, g, h, k \in$ $H\left(D^{n}\right)$, we let

$$
R_{u, v}(z, a)=(f(z)-f(a))(\overline{k(z)}-\overline{k(a)})-(h(z)-h(a))(\overline{g(z)}-\overline{g(a)})
$$

for $z, a \in D^{n}$. The significance of the function $R_{u, v}$ lies in the fact that the commutator $T_{u} T_{v}-T_{v} T_{u}$ can be expressed as an integral operator given by

$$
\left(T_{u} T_{v}-T_{v} T_{u}\right) \psi(a)=\int_{D^{n}} R_{u, v}(z, a) \overline{K_{a}(z)} \psi(z) d V(z)
$$

for $\psi \in A^{2}$ and $a \in D^{n}$. Recall that the functions $f, g, h$ and $k$ are all in $H^{p}$ and hence in $A^{p}$ for all $p>0$ by Proposition [2.2 In particular, we have $R(\cdot, a) \in$ $L^{2}$ for each fixed $a \in D^{n}$. Thus, the above integral is well defined. The above representation is well known. See, for example, 4] for details on the ball. The same proof works on $D^{n}$.

Finally, we prove that (b) implies (a).

Proof of (b) $\Rightarrow$ (a). Put $R=R_{u, v}$ and $R_{\varphi}=R_{u \circ \varphi, v \circ \varphi}$ for $\varphi \in \Phi$. First, we claim the following:

$$
\lim _{a \rightarrow \partial D^{n}} \inf _{\varphi \in \Phi} \int_{D^{n}}\left|R\left(\varphi_{a}(z), a\right)-R_{\varphi}(z, 0)\right|^{14} d V(z)=0 .
$$

Suppose not. Then there exists a net $\left\{w_{\alpha}\right\}$ such that $w_{\alpha} \rightarrow \partial D^{n}$ and

$$
\inf _{\varphi \in \Phi} \int_{D^{n}}\left|R\left(\varphi_{w_{\alpha}}(z), w_{\alpha}\right)-R_{\varphi}(z, 0)\right|^{14} d V(z)>0
$$

for all $w_{\alpha}$. Now, by taking a subnet if necessary, we may assume $\varphi_{w_{\alpha}} \rightarrow \varphi$ for some $\varphi \in \Phi$. Note that

$$
u \circ \varphi_{w_{\alpha}}-u\left(w_{\alpha}\right) \rightarrow u \circ \varphi-u \circ \varphi(0)
$$

uniformly on every compact subset of $D^{n}$. In particular, since $u$ and $v$ are bounded,

$$
u \circ \varphi_{w_{\alpha}}-u\left(w_{\alpha}\right) \rightarrow u \circ \varphi-u \circ \varphi(0) \text { in } L^{28} .
$$

Now, using the $L^{p}$-boundedness of the Bergman projection $P$ for $p>1$, we have in particular

$$
P\left[u \circ \varphi_{w_{\alpha}}-u\left(w_{\alpha}\right)\right] \rightarrow P[u \circ \varphi-u \circ \varphi(0)] \text { in } L^{28} .
$$


On the other hand, letting $u \circ \varphi=F+\bar{G}$ and $v \circ \varphi=H+\bar{K}$, we see that an application of Proposition 4.1 yields

$$
P\left[u \circ \varphi_{w_{\alpha}}-u\left(w_{\alpha}\right)\right]=f \circ \varphi_{w_{\alpha}}-f\left(w_{\alpha}\right), \quad P[u \circ \varphi-u \circ \varphi(0)]=F-F(0) .
$$

Hence, $f \circ \varphi_{w_{\alpha}}-f\left(w_{\alpha}\right) \rightarrow F-F(0)$ in $L^{28}$. Similarly, we have $k \circ \varphi_{w_{\alpha}}-k\left(w_{\alpha}\right) \rightarrow$ $K-K(0)$ in $L^{28}$. It follows that

$$
\left[f \circ \varphi_{w_{\alpha}}-f\left(w_{\alpha}\right)\right]\left[\overline{k \circ \varphi_{w_{\alpha}}-k\left(w_{\alpha}\right)}\right] \rightarrow[F-F(0)][\overline{K-K(0)}] \quad \text { in } \quad L^{14} .
$$

Also, the same is true for functions $h, g$. Hence, we have

$$
R\left(\varphi_{w_{\alpha}}(\cdot), w_{\alpha}\right)-R_{\varphi}(\cdot, 0) \rightarrow 0 \quad \text { in } \quad L^{14},
$$

which is a contradiction to (5.9). Thus, we have (5.8).

Fix $\varphi \in \Phi$ and $\psi \in A^{2}$. Then $T_{u \circ \varphi}$ and $T_{v \circ \varphi}$ are commuting by assumption. Hence, a simple application of (5.1) yields $T_{u \circ \varphi \circ \varphi_{a}} T_{v \circ \varphi_{\circ} \varphi_{a}}=T_{v \circ \varphi_{\circ} \varphi_{a}} T_{u \circ \varphi_{\circ} \varphi_{a}}$ for $a \in D^{n}$. In particular, we have

$$
\left(T_{u \circ \varphi \circ \varphi_{a}} T_{v \circ \varphi \circ \varphi_{a}}-T_{v \circ \varphi \circ \varphi_{a}} T_{u \circ \varphi \circ \varphi_{a}}\right) \psi(a)=0, \quad a \in D^{n} .
$$

Note that

$$
R_{u \circ \varphi \circ \varphi_{a}, v \circ \varphi \circ \varphi_{a}}(z, a)=R_{u \circ \varphi, v \circ \varphi}\left(\varphi_{a}(z), \varphi_{a}(a)\right)=R_{u \circ \varphi, v \circ \varphi}\left(\varphi_{a}(z), 0\right) .
$$

Thus, (5.7) shows

$$
\int_{D^{n}} \frac{R_{\varphi}\left(\varphi_{a}(z), 0\right)}{\prod_{j=1}^{n}\left(1-a_{j} \bar{z}_{j}\right)^{2}} \psi(z) d V(z)=0 .
$$

It follows from (5.7) again that

$$
\left(T_{u} T_{v}-T_{v} T_{u}\right) \psi(a)=\int_{D^{n}} \frac{R(z, a)-R_{\varphi}\left(\varphi_{a}(z), 0\right)}{\prod_{j=1}^{n}\left(1-a_{j} \bar{z}_{j}\right)^{2}} \psi(z) d V(z)
$$

for $a \in D^{n}$.

For each $\rho \in(0,1)$, let $M_{\rho}: L^{2} \rightarrow L^{2}$ be the multiplication operator by the characteristic function of $\rho D^{n}$. Here, $\rho D^{n}=\left\{\rho z: z \in D^{n}\right\}$. Then $M_{\rho}$ is compact when restricted to $A^{2}$. Thus, the operator $M_{\rho}\left(T_{u} T_{v}-T_{v} T_{u}\right)$ is also compact. Put

$$
S_{\rho}=\left(1-M_{\rho}\right)\left(T_{u} T_{v}-T_{v} T_{u}\right)
$$

for simplicity. We note from (5.10) that

$$
S_{\rho} \psi(a)=\chi_{\rho}(a) \int_{D^{n}} \frac{R(z, a)-R_{\varphi}\left(\varphi_{a}(z), 0\right)}{\prod_{j=1}^{n}\left(1-\bar{a}_{j} z_{j}\right)^{2}} \psi(z) d V(z), \quad a \in D^{n}
$$

where $\chi_{\rho}=\chi_{D^{n} \backslash \rho D^{n}}$. 
By (2.2) and simple manipulations, one obtains

$$
\begin{aligned}
\int_{D^{n}} & \frac{\left|R(z, a)-R_{\varphi}\left(\varphi_{a}(z), 0\right)\right|^{2}}{\prod_{j=1}^{n}\left|1-a_{j} \bar{z}_{j}\right|^{2} \sqrt{1-\left|z_{j}\right|^{2}}} d V(z) \\
= & \int_{D^{n}} \frac{\left|R\left(\varphi_{a}(z), a\right)-R_{\varphi}(z, 0)\right|^{2}\left|k_{a}(z)\right|^{2}}{\prod_{j=1}^{n}\left|1-a_{j} \overline{\varphi_{a_{j}}\left(z_{j}\right)}\right|^{2} \sqrt{1-\mid \varphi_{a_{j}}\left(\left.z_{j}\right|^{2}\right.}} d V(z) \\
= & \frac{1}{\prod_{j=1}^{n} \sqrt{1-\left|a_{j}\right|^{2}}} \int_{D^{n}} \frac{\left|R\left(\varphi_{a}(z), a\right)-R_{\varphi}(z, 0)\right|^{2}}{\prod_{j=1}^{n}\left|1-a_{j} \bar{z}_{j}\right| \sqrt{1-\left|z_{j}\right|^{2}}} d V(z) \\
\leq & \frac{1}{\prod_{j=1}^{n} \sqrt{1-\left|a_{j}\right|^{2}}}\left(\int_{D^{n}}\left|R\left(\varphi_{a}(z), a\right)-R_{\varphi}(z, 0)\right|^{14} d V(z)\right)^{1 / 7} \\
& \quad \times\left(\int_{D^{n}} \frac{d V(z)}{\prod_{j=1}^{n}\left|1-a_{j} \bar{z}_{j}\right|^{7 / 6}\left(1-\left|z_{j}\right|^{2}\right)^{7 / 12}}\right)^{6 / 7}
\end{aligned}
$$

where the inequality holds by Hölder's inequality with the conjugate exponents $7 / 6$ and 7. On the other hand, by an application of Lemma 4.2 of [18], we can see

$$
\int_{D^{n}} \prod_{j=1}^{n} \frac{1}{\left|1-a_{j} \bar{z}_{j}\right|^{7 / 6}\left(1-\left|z_{j}\right|^{2}\right)^{7 / 12}} d V(z) \leq C
$$

for some constants $C$. Here and in the rest of the proof, we use the same letter $C$ for various constants depending only on $n$. It follows that

$$
\begin{aligned}
\int_{D^{n}} & \frac{\left|R(z, a)-R_{\varphi}\left(\varphi_{a}(z), 0\right)\right|^{2}}{\prod_{j=1}^{n}\left|1-a_{j} \bar{z}_{j}\right|^{2} \sqrt{1-\left|z_{j}\right|^{2}}} d V(z) \\
& \leq \frac{C}{\prod_{j=1}^{n} \sqrt{1-\left|a_{j}\right|^{2}}}\left(\int_{D^{n}}\left|R\left(\varphi_{a}(z), a\right)-R_{\varphi}(z, 0)\right|^{14} d V(z)\right)^{1 / 7} .
\end{aligned}
$$

Now, the Cauchy-Schwarz inequality yields

$$
\begin{aligned}
\left|S_{\rho} \psi(a)\right|^{2} \leq & \left(\chi_{\rho}(a) \int_{D^{n}} \frac{\left|R(z, a)-R_{\varphi}\left(\varphi_{a}(z), 0\right)\right|}{\prod_{j=1}^{n}\left|1-a_{j} \bar{z}_{j}\right|^{2}}|\psi(z)| d V(z)\right)^{2} \\
\leq & \left(\int_{D^{n}} \frac{\chi_{\rho}(a)\left|R(z, a)-R_{\varphi}\left(\varphi_{a}(z), 0\right)\right|^{2}}{\prod_{j=1}^{n}\left|1-a_{j} \bar{z}_{j}\right|^{2} \sqrt{1-\left|z_{j}\right|^{2}}} d V(z)\right) \\
& \times\left(\int_{D^{n}} \prod_{j=1}^{n} \frac{\sqrt{1-\left|z_{j}\right|^{2}}}{\left|1-a_{j} \bar{z}_{j}\right|^{2}}|\psi(z)|^{2} d V(z)\right) \\
\leq & C \frac{\chi_{\rho}(a)}{\prod_{j=1}^{n} \sqrt{1-\left|a_{j}\right|^{2}}\left(\int_{D^{n}}\left|R\left(\varphi_{a}(z), a\right)-R_{\varphi}(z, 0)\right|^{14} d V(z)\right)^{1 / 7}} \\
& \times\left(\int_{D^{n}} \prod_{j=1}^{n} \frac{\sqrt{1-\left|z_{j}\right|^{2}}}{\left|1-a_{j} \bar{z}_{j}\right|^{2}}|\psi(z)|^{2} d V(z)\right) .
\end{aligned}
$$


It follows from Fubini's theorem that

$$
\begin{array}{rl}
\int_{D^{n}}\left|S_{\rho} \psi\right|^{2} & d V \\
\leq & \sup _{a \in D^{n} \backslash \rho D^{n}}\left(\int_{D^{n}}\left|R\left(\varphi_{a}(z), a\right)-R_{\varphi}(z, 0)\right|^{14} d V(z)\right)^{1 / 7} \\
& \times \int_{D^{n}} \prod_{j=1}^{n} \sqrt{1-\left|z_{j}\right|^{2}}|\psi(z)|^{2} \int_{D^{n}} \frac{d V(a)}{\prod_{j=1}^{n}\left|1-a_{j} \bar{z}_{j}\right|^{2} \sqrt{1-\left|a_{j}\right|^{2}}} d V(z) .
\end{array}
$$

Moreover, by an application of Lemma 4.2 .2 of [18], we have

$$
\int_{D^{n}} \frac{d V(a)}{\prod_{j=1}^{n}\left|1-a_{j} \bar{z}_{j}\right|^{2} \sqrt{1-\left|a_{j}\right|^{2}}} \leq \frac{C}{\prod_{j=1}^{n} \sqrt{1-\left|z_{j}\right|^{2}}}, \quad z \in D^{n},
$$

and therefore, we have

$$
\int_{D^{n}}\left|S_{\rho} \psi\right|^{2} d V \leq C \sup _{a \in D^{n} \backslash \rho D^{n}}\left(\int_{D^{n}}\left|R\left(\varphi_{a}(z), a\right)-R_{\varphi}(z, 0)\right|^{14} d V(z)\right)^{1 / 7} \int_{D^{n}}|\psi|^{2} d V .
$$

Note that the above holds for all $\varphi \in \Phi$ and $\psi \in A^{2}$. So, we finally have

$$
\left\|S_{\rho}\right\| \leq C \sup _{a \in D^{n} \backslash \rho D^{n}}\left\{\inf _{\varphi \in \Phi} \int_{D^{n}}\left|R\left(\varphi_{a}(z), a\right)-R_{\varphi}(z, 0)\right|^{14} d V(z)\right\}^{1 / 14} .
$$

Now, taking the limit $\rho \rightarrow 1$, we conclude $S_{\rho} \rightarrow 0$ in the operator norm by (5.8). Hence, $T_{u} T_{v}-T_{v} T_{u}$ can be approximated by compact operators, so it is compact, as desired. The proof is complete.

We say that a bounded linear operator $L$ on a Hilbert space is essentially normal if $L$ and its adjoint operator are essentially commuting. As a consequence of Theorem 1.3 and Corollary 4.2, we have the following.

Corollary 5.2. Let $u \in L^{\infty}$ be a pluriharmonic symbol and assume $u=f+\bar{g}$ for some $f, g \in H\left(D^{n}\right)$. Then, the following statements are equivalent:

(a) $T_{u}$ is essentially normal on $A^{2}$.

(b) $|f|^{2}-|g|^{2}$ is boundary $n$-harmonic.

(c) For each $\varphi \in \Phi$, there are pairwise disjoint sets $I_{1}, \ldots, I_{m}$ with $\bigcup_{j=1}^{m} I_{j}=I$ for some positive integer $m \leq n$, functions $p_{1}, \ldots, p_{m}$ with $p_{j} \in H\left(I_{j}\right)$ for each $j$, and unimodular constants $\alpha_{1}, \ldots, \alpha_{m}$ such that

$$
u \circ \varphi=\sum_{j=1}^{m} \alpha_{j}\left(p_{j}+\bar{p}_{j}\right)+\lambda
$$

for some constant $\lambda$.

As a consequence of Theorem 1.4, Corollary 4.2 and Corollary 5.2 , we obtain the following.

Corollary $5.3(n \geq 3)$. Let $u \in L^{\infty}$ be a pluriharmonic symbol and assume $u=f+\bar{g}$ for some $f, g \in H\left(D^{n}\right)$. Then, the following statements are equivalent:

(a) $T_{u}$ is normal on $A^{2}$.

(b) $T_{u}$ is essentially normal on $A^{2}$.

(c) $|f|^{2}-|g|^{2}$ is n-harmonic.

(d) $|f|^{2}-|g|^{2}$ is boundary $n$-harmonic. 
Also, as an immediate consequence of Theorem 1.1. Theorem 1.3 and Corollary 3.6, we recover the result of Sun and Zheng [14] mentioned in the Introduction.

Corollary $5.4(n \geq 2)$. Let $f, g \in H^{\infty}$. Then, the following statements are equivalent:

(a) $T_{f} T_{\bar{g}}=T_{\bar{g}} T_{f}$ on $A^{2}$.

(b) For each $j$, we have either $\partial_{j} f=0$ or $\partial_{j} g=0$.

(c) $T_{f}$ and $T_{\bar{g}}$ are essentially commuting on $A^{2}$.

Example. Corollary 5.3 does not extend to $n=2$, either. To see an example, let

$$
f(z)=\sum_{\ell=0}^{\infty} a_{\ell}\left(\sum_{i+j=\ell} z_{1}^{i} z_{2}^{j}\right), \quad z=\left(z_{1}, z_{2}\right),
$$

where coefficients $a_{\ell} \neq 0$ are chosen so that the series converges on all of $\mathbb{C}^{2}$. Then, a little manipulation yields

$$
z_{1} f(z)=\sum_{\ell=0}^{\infty} a_{\ell+1}\left(\sum_{i+j=\ell} z_{1}^{i+1} z_{2}^{j+1}\right)+\psi\left(z_{1}\right),
$$

where $\psi(\lambda)=\sum_{\ell=0}^{\infty} a_{\ell} \lambda^{\ell+1}$. Define $g(z)=z_{1} f(z)-\psi\left(z_{1}\right)$. By symmetry, we have $g(z)=z_{2} f(z)-\psi\left(z_{2}\right)$. Hence, we have $\partial_{1} g(z)=z_{2} \partial_{1} f(z), \partial_{2} g(z)=z_{1} \partial_{2} f(z)$ and thus

$$
\widetilde{\Delta}_{j}\left(|f|^{2}-|g|^{2}\right)(z)=\left(1-\left|z_{j}\right|^{2}\right)\left(1-\left|z_{1}\right|^{2}\right)\left(1-\left|z_{2}\right|^{2}\right)\left|\partial_{j} f(z)\right|^{2}
$$

for $j=1,2$. Consequently, $|f|^{2}-|g|^{2}$ is boundary 2-harmonic, but not 2-harmonic.

\section{Semi-commuting Toeplitz operators}

For Toeplitz operators $T_{u}$ and $T_{v}$, we call $T_{u} T_{v}-T_{u v}$ the semi-commutator. For Toeplitz operators with pluriharmonic symbols, the commuting property is very closely related to the semi-commuting property.

To see what is going on, let us begin with functions $f, g, h, k \in H^{\infty}$. Put $u=f+\bar{g}$ and $v=h+\bar{k}$. Then, one can easily verify that

$$
T_{u} T_{v}-T_{u v}=T_{f} T_{\bar{k}}-T_{\bar{k}} T_{f}
$$

Hence, the semi-commuting problem of $T_{u}$ and $T_{v}$ simply reduces to the commuting problem of $T_{f}$ and $T_{\bar{k}}$. Thus, for $n \geq 2$, the essentially semi-commuting property is the same as the semi-commuting property by Corollary 5.4 .

For general pluriharmonic symbols, our arguments used in this paper can be easily modified to conclude the same. Lemma 6.1 and Lemma 6.2 below are valid even for $n=1$. For other characterizations on the disk and ball, see [15] and [16].

Lemma 6.1. Let $u, v \in L^{\infty}$ be pluriharmonic symbols and assume $u=f+\bar{g}, v=$ $h+\bar{k}$ for some $f, g, h, k \in H\left(D^{n}\right)$. Then, the following statements are equivalent:

(a) $T_{u} T_{v}=T_{u v}$ on $A^{2}$.

(b) For each $j$, we have either $\partial_{j} f=0$ or $\partial_{j} k=0$.

Proof. As in the proof Theorem 1.1, one obtains

$$
\left(T_{u} T_{v}-T_{u v}\right) k_{a}=f \overline{k(a)} k_{a}-P\left(f \bar{k} k_{a}\right) .
$$

Thus, $T_{u} T_{v}=T_{u v}$ if and only if

$$
P\left(f \bar{k} k_{a}\right)=f \overline{k(a)} k_{a},
$$


which is in turn equivalent to the fact that $\partial_{j} f=0$ or $\partial_{j} k=0$ for each $j$ by Proposition 4.1, because $f, k \in H^{2}$ by Proposition 2.2 The proof is complete.

For essentially semi-commuting Toeplitz operators, we also have the following.

Lemma 6.2. Let $u, v \in L^{\infty}$ be pluriharmonic symbols and assume $u=f+\bar{g}, v=$ $h+\bar{k}$ for some $f, g, h, k \in H\left(D^{n}\right)$. Then, the following statements are equivalent:

(a) $T_{u} T_{v}-T_{u v}$ is compact on $A^{2}$.

(b) $T_{u \circ \varphi} T_{v \circ \varphi}=T_{(u v) \circ \varphi}$ on $A^{2}$ for every $\varphi \in \Phi$.

(c) $f \bar{k}$ is boundary n-harmonic.

Proof. As in (5.7), we have the following representation:

$$
\left(T_{u} T_{v}-T_{u v}\right) \psi(a)=\int_{D^{n}}(f(z)-f(a))(\overline{k(z)}-\overline{k(a)}) \overline{K_{a}(z)} \psi(z) d V(z)
$$

for $a \in D^{n}$ and $\psi \in A^{2}$. Hence, one can easily modify the proof of Theorem 1.3 to conclude the theorem. The proof is complete.

Now, combining Corollary 3.6 Lemma 6.1 and Lemma 6.2 we see that the essentially semi-commuting property is the same as the semi-commuting property for $n \geq 2$.

Theorem $6.3(n \geq 2)$. Let $u, v \in L^{\infty}$ be pluriharmonic symbols and assume $u=f+\bar{g}, v=h+\bar{k}$ for some $f, g, h, k \in H\left(D^{n}\right)$. Then, the following statements are equivalent:

(a) $T_{u} T_{v}=T_{u v}$ on $A^{2}$.

(b) For each $j$, we have either $\partial_{j} f=0$ or $\partial_{j} k=0$.

(c) $T_{u} T_{v}-T_{u v}$ is compact on $A^{2}$.

(d) $T_{u \circ \varphi} T_{v \circ \varphi}=T_{(u v) \circ \varphi}$ on $A^{2}$ for every $\varphi \in \Phi$.

(e) $f \bar{k}$ is boundary n-harmonic.

\section{REFERENCES}

[1] S. Axler and Z̆. Cučković, Commuting Toeplitz Operators with Harmonic Symbols, Integr. Equat. Oper. Th. 14 (1991), 1-11. MR 92f:47018

[2] S. Axler and Z̆. Cučcković, and N. V. Rao, Commutants of Analytic Toeplitz Operators on the Bergman Space, Proc. Amer. Math. Soc. 128 (2000), 1951-1953. MR 2000m:47035

[3] B. R. Choe and Y. J. Lee, Pluriharmonic Symbols of Commuting Toeplitz Operators, Illinois J. Math. 37 (1993), 424-436. MR 94i:47041

[4] B. R. Choe and Y. J. Lee, Pluriharmonic Symbols of Essentially Commuting Toeplitz Operators, Illinois J. Math. 42 (1998), 280-293. MR 99g:47051

[5] B. R. Choe and Y. J. Lee, Commuting Toeplitz Operators on the Harmonic Bergman Space, Michigan Math. J. 46 (1999), 163-174. MR 2000a:47054

[6] Z̆. Cučcković, Commuting Toeplitz Operators on the Bergman Space of an Annulus, Michigan Math. J. 43 (1996), 355-365. MR 97k:47026

[7] S. G. Krantz, Function Theory of Several Complex Variables, John Wiley \& Sons, New York, 1982. MR 84c:32001

[8] J. Lee, An Invariant Mean Value Property in the Polydisc, Illinois J. Math. 42 (1998), 406419. MR 99k:31003

[9] Y. J. Lee Pluriharmonic Symbols of Commuting Toeplitz Type Operators on the Weighted Bergman Spaces, Canadian Math. Bull. 41 (1998), 129-136. MR 99b:47035]

[10] Y. J. Lee and K. Zhu, Some Differential and Integral Equations with Applications to Toeplitz Operators, Integr. Equat. Oper. Th. 44 (2002), 466-479.

[11] W. Rudin, Function Theory in Polydiscs, W. A. Benjamin, 1969. MR 41:501

[12] W. Rudin, Functional Analysis, McGraw-Hill, 1973. MR 51:1315] 
[13] K. Stroethoff, Essentially Commuting Toeplitz Operators with Harmonic Symbols, Canadian J. Math. 45 (1993), 1080-1093. MR 94h:47046

[14] S. Sun and D. Zheng, Toeplitz Operators on the Polydisk, Proc. Amer. Math. Soc. 124 (1996), 3351-3356. MR 97a:47038

[15] D. Zheng, Hankel Operators and Toeplitz Operators on the Bergman Space, J. Functional Anal. 83 (1989), 98-120. MR 91b:47057

[16] D. Zheng, Semi-commutators of Toeplitz Operators on the Bergman Space, Integr. Equat. Oper. Th. 25 (1996), 347-372. MR 97e:47039

[17] D. Zheng, Commuting Toeplitz Operators with Pluriharmonic Symbols, Trans. Amer. Math. Soc. 350 (1998), 1595-1618. MR 98i:47027

[18] K. Zhu, Operator Theory in Function Spaces, Marcel Dekker. New York and Basel, 1990. MR 92c: 47031

Department of Mathematics, Korea University, Seoul 136-701, Korea

E-mail address: choebr@math.korea.ac.kr

Department of Mathematics, Korea University, Seoul 136-701, Korea

E-mail address: koohw@math.korea.ac.kr

Department of Mathematics, Mokpo National University, Chonnam 534-729, Korea

E-mail address: yjlee@mokpo.ac.kr 